\title{
On properties of turbulence models
}

\author{
T. H. Moulden \\ The University of Tennessee Space Institute, USA
}

\begin{abstract}
Many applications of fluid mechanics are to turbulent flows. The practical computation of such flows call upon turbulence models to provide the information needed to close the mean motion equations. The present comments concern those properties of turbulence models that may be obtained by direct study of the field equations defining these models. The second moment model and the $k \sim \epsilon$ model are the focus of attention and norm inequalities are written for these models. The stability properties of the associated dynamical system are determined. Some difficulties associated with obtaining norm estimates are also discussed.
\end{abstract}

Keywords: turbulence models, energy estimates.

\section{Background}

It is generally accepted that the Navier-Stokes equations describe the motion of fluids under normal conditions of pressure and temperature. It is also accepted that these same equations describe turbulent fluid motion (the "sinuous motion" described by Stokes and studied by Reynolds). A caution is necessary at this point since the consistency of the Navier-Stokes equations has not been documented. In particular, there is no complete existence, uniqueness and regularity results available for these equations when provided with general boundary conditions.

Restrict attention to constant density fluid motion. Due to the lack of a complete theory for the constant density Navier-Stokes equations:

$$
\begin{gathered}
\frac{\partial v_{i}}{\partial x_{i}}=0 \\
\frac{\partial v_{i}}{\partial t}+\frac{\partial\left(v_{i} v_{j}\right)}{\partial x_{j}}+\frac{\partial p}{\partial x_{i}}=\nu \nabla^{2}\left(v_{i}\right)+f_{i}
\end{gathered}
$$

noted above, it cannot be expected that a complete theory exists for the mean motion equations used to treat turbulent flow. Here $\mathbf{v}(\mathbf{x}, t)$ is the instantaneous 
velocity field and $p(\mathbf{x}, t)$ the corresponding pressure field (normalized by the constant fluid density). Turbulence models have, as a prequel, the existence of a Reynolds decomposition $\mathbf{v} \mapsto \mathbf{V}+\mathbf{u}$ where $\mathbf{V}=\mathcal{E}(\mathbf{v})$, denotes the mean velocity, and $\mathbf{u}(\mathbf{x}, t)$ a fluctuating velocity field. The resulting lack of closure found in the mean motion equations requires that a turbulence model be included. While such a model closes the field equations, it is not based upon any real physical principle and contains large empirical and heuristic content.

A turbulence model is said to be meaningful if it produces a closed set of mean motion equations for which a unique solution exists. This definition makes no mention of the predictive power of the turbulence model: that is a separate issue and not addressed herein (but see Gatski [1] for a discussion of turbulence models). Rather, the present interest focuses upon the mathematical properties of such models with special reference to energy estimates. The standard energy estimate for equations (1) and (2) gives:

$$
|\mathbf{v}|_{g}^{2}(t) \leq|\mathbf{f}|_{g}^{2} /\left(\nu L_{v}^{2}\right)^{2} \quad \text { as } t \rightarrow \infty
$$

when a body force, $\mathbf{f}(\mathbf{x})$, is present but independent of time. The global norm of some vector $\mathbf{p},|\mathbf{p}|_{g}^{2}$, is defined to be $\int_{\mathcal{D}}\langle\mathbf{p}, \mathbf{p}\rangle d V$ where the standard inner product $\langle\mathbf{p}, \mathbf{p}\rangle \equiv p_{i} p_{i}$ (sum on $i$ ) is defined. $\mathcal{D}$ denotes the (bounded) domain occupied by the fluid with $\partial \mathcal{D}$ its boundary. In the absence of body forces, the velocity norm $|\mathbf{v}|_{g}^{2}(t)$, decays exponentially (see Foias et al. [2], for example):

$$
|\mathbf{v}|_{g}^{2}(t) \leq \exp \left[-\nu L_{v}^{2} t\right]|\mathbf{v}|_{g}^{2}(t=0)
$$

as $t \rightarrow \infty$. Here $L_{v}$ is the constant in a Poincaré inequality of the form $\|\mathbf{L}\|_{g}^{2} \geq$ $L_{v}^{2}|\mathbf{v}|_{g}^{2}$. These results hold for the instantaneous Navier-Stokes equations and hence apply to both laminar and turbulent flows. When the Reynolds decomposition is applied to the Navier-Stokes equations and the mean motion equations obtained, it follows that the same bounds on $|\mathbf{v}|_{g}$ must hold (but now $\mathbf{v}=\mathbf{V}+\mathbf{u}$ implies that $|\mathbf{v}|_{g} \leq|\mathbf{V}|_{g}+|\mathbf{u}|_{g}$ and a direct comparison between $|\mathbf{v}|_{g}$ and $|\mathbf{V}|_{g}$ is not possible). A valid turbulence model must produce a norm $|\mathbf{V}|_{g}$ that is consistent in properties with these estimates for the instantaneous equations: that is, $|\mathbf{V}|_{g}$ must decay exponentially as $t \rightarrow \infty$ in the absence of a body force. For example, it was shown in Moulden [3] that the classical Boussinesq model has this property simply because, in norm, it has the effect of augmenting the fluid viscosity as $\nu \mapsto \nu+\nu_{\tau}$ for some eddy viscosity coefficient $\nu_{\tau}$. As such the Boussinesq model does not reveal any physics associated with fluid turbulence other than an enhanced rate of dissipation.

The first axiom of Newtonian mechanics provides the foundation for the symmetry structure of the equations of fluid mechanics. That is, the equations must be covariant under the Galilean transformation group $\mathbb{G}_{a}$. All constructs in the theory must respect this symmetry. It is well known that the mean velocity field transforms as $\mathbf{V} \mapsto \mathbf{Q V}+\mathbf{V}_{B}$ under $\mathbb{G}_{a}$ while the fluctuating velocity has the transformation $\mathbf{u} \mapsto \mathbf{Q u}$. The Reynolds tensor $\mathcal{R}$ is frame indifferent under $\mathbb{G}_{a}$. Here $\mathbf{Q}$ is a constant orthogonal matrix and $\mathbf{V}_{B}$ the constant Galilean boost velocity. Details of these transformations are given in Speziale [4]. It follows directly that 
both $\operatorname{div}(\mathbf{V}) \mapsto \operatorname{div}(\mathbf{V})$ and $\operatorname{div}(\mathbf{u}) \mapsto \operatorname{div}(\mathbf{u})$ : the mean and fluctuating continuity equations are both covariant under the transformation group $\mathbb{G}_{a}$. Turbulence models must be consistent with these transformations.

The mean motion equations can be extracted from the instantaneous equations (1) and (2) by means of the standard Reynolds decomposition noted above to give the system:

$$
\begin{gathered}
\frac{\partial V_{i}}{\partial x_{i}}=0 \\
\frac{\partial V_{i}}{\partial t}+\frac{\partial\left(V_{i} V_{j}\right)}{\partial x_{j}}+\frac{\partial \mathcal{R}_{i j}}{\partial x_{j}}+\frac{\partial P}{\partial x_{i}}=\nu \nabla^{2}\left(V_{i}\right)+f_{i}
\end{gathered}
$$

In equation (4), $P$ denotes the mean pressure field (again normalized by the fluid density) and $\mathbf{f}(\mathbf{x})$ a time independent body force. A turbulence model is taken to be a statement of the form:

$$
\phi(\mathcal{R}, \mathbf{V}, \boldsymbol{\alpha})=0
$$

if $\mathcal{R} \equiv \mathcal{E}(\mathbf{u} \otimes \mathbf{u})$ denotes the Reynolds tensor. The function $\phi$ may be vector valued and may include partial derivatives as a set of evolutionary equations. $\phi$ is referred to as a representation of a turbulent flow. $\alpha$ represents a vector of model constants whose values are usually determined empirically. While equation (5) may close the set of equations (3) and (4), it leaves many questions unresolved. It is not known what, if any, bounds constrain the set of constants $\left\{\alpha_{i}\right\}$ for a given model to posses a stable physically realistic solution.

It is not clear, for example, that equations (3) and (4) have a solution for any arbitrary model contained in equation (5). This question is more fundamental than asking for equation (5) to reproduce the properties of turbulent flows since the existence of a unique solution is a pre-requisite for predictive performance. Formally:

\section{"A meaningful turbulence model is one for which the system of equations (3), (4), (5) possesses a unique regular solution. In addition, the system should have stable fixed points and must be covariant under the group $\mathbb{G}_{a}$."}

No test is available to ascertain if equation (5) does, in fact, specify a meaningful turbulence model. The objective herein is to present the information that can be obtained from energy estimates for the equations of turbulence models (as introduced in Moulden [3] for a second moment closure model). A few comments are made about a second moment model and the well-known $k \sim \epsilon$ model is also considered.

Restricting attention to "meaningfulness" does not remove the importance of the criteria written by Hanjalić [5] for the construction of turbulence models. In particular: the model should satisfy the realizability constraints (the turbulence kinetic energy must be non-negative for example) and be computationally manageable. It must also satisfy the invariance requirements consistent with Newtonian continuum mechanics. That is, more is involved in the construction of a turbulence model than just the heuristic arguments required to select the functional form of modeled 
terms. The norm estimates discussed below should be attended to at the same time as other computational issues are considered. Assessing the role of model constants in the stability properties of the model should be an essential feature of model development.

\section{A uniqueness theorem}

A uniqueness theorem for the mean motion equations, that follows the work of Gurtin [6], can be written for equations (3) and (4) without appeal to a turbulence model of the form given in equation (5). This result may be stated as:

Lemma I. Let $\left(\mathbf{V}_{1}, \bar{P}_{1}\right)$ and $\left(\mathbf{V}_{2}, \bar{P}_{2}\right)$ be two solutions of the mean motion equations in (3) and (4) (with the same boundary conditions and body forces). Then:

$$
\mathbf{V}_{1}=\mathbf{V}_{2} ; \quad \mathcal{R}_{1}+\bar{P}_{1} \mathbf{I}=\left[\boldsymbol{\mathcal { R }}_{2}+\mathcal{C}(\mathbf{x}, t)\right]+\left[\bar{P}_{2}+Q(\mathbf{x}, t)\right] \mathbf{I}
$$

such that the quantity $\mathcal{C}(\mathbf{x}, t)$ is an arbitrary divergence-free symmetric second order tensor and $Q$ an arbitrary scalar field whose spatial gradient $\nabla(Q)$, vanishes $Q=Q(t)$ only.

and is valid provided that the regularity required by the proof holds. The result shows that while the mean velocity field is specified uniquely by the mean motion equations, the Reynolds tensor need not be unique. The same is true of the mean pressure field. The proof of this lemma, being given in Moulden [7], need not be repeated here. The lemma implies that equation (5) cannot define a unique representation, $\phi$, of any particular turbulent flow model. However, the non-uniqueness in $\mathcal{R}$ is of the form $\mathcal{R} \mapsto \mathcal{R}+\mathcal{C}$ with $\operatorname{div}(\mathcal{C})=\mathbf{0}$ so that the computation of the mean velocity is not compromised in equations (3) and (4). Such a finding is on the same footing as noticing that the mass invariance constraint $\operatorname{div}(\mathbf{u})=0$ allows a gauge field, $\mathbf{w}(\mathbf{x}, t)$, from the condition $\operatorname{div}(\mathbf{u}+\mathbf{w})=0$ iff $\operatorname{div}(\mathbf{w})=0$. The role of gauge fields in turbulence models will be addressed elsewhere and can be adopted to describe the inactive component of the motion. For a discussion of this inactive motion, and other problems related to turbulence, see for example, Bradshaw [9].

In the proof of Lemma I it must be assumed that the mean velocity gradient $\overline{\mathbf{L}}=\partial \mathbf{V} / \partial \mathbf{x}$ has bounded eigenvalues (but, there is no proof that this assumed regularity is true). The local regularity results (ones that do not consider the influence of the initial conditions) for the Navier-Stokes equations are discussed in Seregin [8]. Of course, it is the non-linear terms that impede the progress in constructing a full theory. It is known that if the boundary data is smooth on a bounded domain then there is an "almost everywhere" restriction on solutions: sets of measure zero may contain singular behaviour. It is not clear how such a theory can be extended to the mean motion equations, (3) and (4), due to the lack of information about the properties of the Reynolds tensor, $\mathcal{R}$, as defined by the turbulence model given in equation (5). The second moment equation has little to offer here due to the lack of closure of that equation (the $\Psi$ term of equation (8) below must be 
represented by a turbulence model). The present approach assumes sufficient regularity to obtain the results for the selected turbulence model: these assumptions are, however, stated as the discussion proceeds.

\section{Energy estimates}

Energy estimates start from the definition of norm quantities such as the velocity $|\mathbf{V}|^{2}=\langle\mathbf{V}, \mathbf{V}\rangle$ and its global extension $|\mathbf{V}|_{g}^{2} \equiv \int_{\mathcal{D}}\langle\mathbf{V}, \mathbf{V}\rangle d V$. The latter of which can be evaluated from the norm energy equation for the mean velocity:

$$
\frac{1}{2} \frac{\partial}{\partial t}|\mathbf{V}|_{g}^{2}+\nu\|\overline{\mathbf{L}}\|_{g}^{2}=\langle\overline{\mathbf{L}}, \boldsymbol{R}\rangle_{g}+\langle\mathbf{V}, \mathbf{f}\rangle_{g}
$$

a result obtained from equation (4) by means of an inner product with the mean velocity. Periodic boundary conditions have been assumed for this estimate and will be adopted throughout the following. The standard Poincaré inequality allows the statement $\|\overline{\mathbf{L}}\|_{g}^{2} \geq L_{L}^{2}|\mathbf{V}|_{g}^{2}$ to replace derivative norms by value norms. Hence there is the differential inequality:

$$
\frac{\partial}{\partial t}|\mathbf{V}|_{g}^{2}+2 \nu L_{L}^{2}|\mathbf{V}|_{g}^{2} \leq 2\|\overline{\mathbf{L}}\|_{g}\|\boldsymbol{\mathcal { R }}\|_{g}+2|\mathbf{V}|_{g}|\mathbf{f}|_{g}
$$

$\overline{\mathbf{L}}$ denotes the mean motion velocity gradient $\partial \mathbf{V} / \partial \mathbf{x}$. Herein, the notation $\langle\mathbf{A}, \mathbf{B}\rangle_{g}=\int_{\mathcal{D}} \operatorname{trace}\left(\mathbf{A B}^{T}\right) d V$ is adopted for the global inner product of any pair of linear operators, $\mathbf{A}$ and $\mathbf{B}$, on $\mathbb{R}^{3}$. Locally, $\langle\mathbf{A}, \mathbf{B}\rangle=\operatorname{trace}\left(\mathbf{A B}^{T}\right)$. Equation (6) requires an estimate for $\|\mathcal{R}\|_{g}$ before anything can be said about the evolution of the mean velocity norm. This estimate must be prepared for each turbulence model considered. A class of invariant models were studied in Moulden [3] with the conclusion that a stable system was only possible for a restricted parameter vector $\boldsymbol{\alpha}$ in equation (5). A brief revue of such a model is included here to illustrate the problems involved.

It is important to recognize that regularity assumptions are included in equation (6) in that the norm $\|\overline{\mathbf{L}}\|_{g}^{2}=\int_{\mathcal{D}} \operatorname{trace}\left(\overline{\mathbf{L}} \overline{\mathbf{L}}^{T}\right) d V$ and the global inner product $\langle\mathcal{R}, \overline{\mathbf{L}}\rangle_{g}$ are both assumed to be bounded (which demands that both $\langle\mathcal{R}, \overline{\mathbf{L}}\rangle$ and $\langle\overline{\mathbf{L}}, \overline{\mathbf{L}}\rangle$ be bounded everywhere and for all time). This requirement can be expressed as the need for the eigenvalues of both $\overline{\mathbf{L}}$ (a property of the mean motion) and $\mathcal{R}$ (a property of the velocity fluctuations) to be bounded everywhere and for all time. The Cauchy-Schwarz inequality provides an upper bound:

$$
\langle\mathcal{R}, \overline{\mathbf{L}}\rangle_{g} \leq\|\mathcal{R}\|_{g}\|\overline{\mathbf{L}}\|_{g} \leq\left[\|\mathcal{R}\|_{g}^{2}+\|\overline{\mathbf{L}}\|_{g}^{2}\right] / 2
$$

This inequality and the Poincare inequality allow inequality (6) to be expressed in the form:

$$
\frac{\partial}{\partial t}|\mathbf{V}|_{g}^{2}+\nu L_{L}^{2}|\mathbf{V}|_{g}^{2} \leq \frac{2}{\nu}\|\boldsymbol{R}\|_{g}^{2}+\frac{2}{\nu L_{L}^{2}}|\mathbf{f}|_{g}^{2}
$$

to show the role that the norms of the Reynolds tensor and body force play in the stability of the mean motion. If regularity of the mean motion is assumed to the 
extent that the mean velocity gradient, $\overline{\mathbf{L}}$, and its temporal and spatial gradients exist then equation (4) gives:

$$
\frac{d \overline{\mathbf{L}}}{d t}+\overline{\mathbf{L}}^{2}+\nabla(\operatorname{div}(\mathcal{R}))=\nu \nabla^{2}(\overline{\mathbf{L}})-\mathbf{P}+\mathbf{F}
$$

where $\mathbf{F}=\boldsymbol{\nabla}(\mathbf{f})$ and $\mathbf{P}$ denotes the mean motion pressure Hessian. Both of which are assumed to exist. Then an energy equation for the mean velocity gradient emerges in the form:

$$
\frac{\partial}{\partial t}\|\overline{\mathbf{L}}\|_{g}^{2}+2 \nu\|\nabla \overline{\mathbf{L}}\|_{g}^{2}+2\left\langle\langle\mathbf{K}, \overline{\mathbf{L}}\rangle_{g}=2\left\langle\langle\mathbf{F}, \overline{\mathbf{L}}\rangle_{g}\right.\right.
$$

if $\mathbf{K}=\overline{\mathbf{L}}^{2}+\nabla(\operatorname{div}(\mathcal{R}))$.

It was shown in Moulden [3] that there is no unique estimate available for the Reynolds stress models of the form discussed by Lewellen [10]. One reason for the lack of a unique estimate is the existence of a norm hierarchy for the mean motion equations. Estimates for turbulence models can use zero order norm closer based upon the level of equation (6), or can have first order norm closure using equation (7) with its additional regularity assumptions. In both cases conditional linear stability (depending upon model constants) was found at the origin of the phase space of the respective dynamical systems when body forces are absent. Physical significance of the estimates also needs to be considered. The process is illustrated below for zero order norm closure.

Second moment closure models are based upon the system of equations defined by:

$$
\frac{\partial \mathcal{R}_{i j}}{\partial t}+V_{k} \frac{\partial \mathcal{R}_{i j}}{\partial x_{k}}+\mathcal{R}_{j k} \frac{\partial V_{i}}{\partial x_{k}}+\mathcal{R}_{i k} \frac{\partial V_{j}}{\partial x_{k}}-\nu \frac{\partial^{2} \mathcal{R}_{i j}}{\partial x_{k}^{2}}=-\Psi_{i j}
$$

(a set of six equations) for the Reynolds tensor $\mathcal{R}$. Equation (8) makes use of the notation:

$$
\Psi_{i j}=2 \nu \mathcal{E}\left[\frac{\partial u_{i}}{\partial x_{k}} \frac{\partial u_{j}}{\partial x_{k}}\right]+\frac{\partial}{\partial x_{k}} \mathcal{E}\left(u_{i} u_{j} u_{k}\right)+\left.\psi_{i j} \equiv \mathbf{E}_{T}\right|_{i j}+\left.\mathbf{D}_{F}\right|_{i j}+\psi_{i j}
$$

with $\mathbf{E}_{T}$ the turbulence dissipation and $\mathbf{D}_{F}$ the velocity diffusion term. The linear combination:

$$
\psi_{i j}=\left[\frac{\partial}{\partial x_{i}}-\mathcal{E}\left(u_{j} p\right)+\frac{\partial}{\partial x_{j}} \mathcal{E}\left(u_{i} p\right)\right]-\left.\mathcal{E}\left[p\left(\frac{\partial u_{j}}{\partial x_{i}}+\frac{\partial u_{i}}{\partial x_{j}}\right)\right] \equiv \mathbf{D}_{P}\right|_{i j}+\left.\mathbf{S}_{P}\right|_{i j}
$$

of the pressure strain and pressure diffusion defines the tensor $\psi_{i j}$. Write the right hand side of equation (8) in the form: $\boldsymbol{\Psi}=\mathbf{E}_{T}+\mathbf{D}_{F}+\mathbf{D}_{P}+\mathbf{S}_{P}$ to allow the estimate:

$$
\frac{\partial}{\partial t}\|\boldsymbol{\mathcal { R }}\|_{g}^{2}+2 \nu\|\boldsymbol{\nabla} \mathcal{R}\|_{g}^{2}+4\langle\overline{\mathbf{L}} \boldsymbol{R}, \mathcal{R}\rangle_{g}+2\left\langle\langle\boldsymbol{\Psi}, \mathcal{R}\rangle_{g}=0\right.
$$

again for periodic boundary conditions. The case of zero order norm closure will illustrate some of the problems associate with the construction of meaningful estimates. The dynamical system for that estimate is given by:

$$
\frac{\partial}{\partial t}\left(\begin{array}{c}
|\mathbf{V}|_{g}^{2} \\
\|\boldsymbol{R}\|_{g}^{2}
\end{array}\right) \leq\left(\begin{array}{c}
-\nu L_{L}^{2}|\mathbf{V}|_{g}^{2}+2\|\mathcal{R}\|_{g}^{2} / \nu+2|\mathbf{f}|_{g}^{2} /\left(\nu L_{L}^{2}\right) \\
-2 \nu L_{R}^{2}|\mathcal{R}|_{g}^{2}-2\left(\nu+\Lambda^{*}-2 \bar{L}\right)\|\mathcal{R}\|_{g}^{2}
\end{array}\right)
$$


and is based upon equations (6a) and (9). The estimate:

$$
\langle\overline{\mathbf{L}} \boldsymbol{\mathcal { R }}, \boldsymbol{\mathcal { R }}\rangle_{g} \leq\|\overline{\mathbf{L}}\|_{g}\|\mathcal{R}\|_{g}^{2} \leq \bar{L}\|\mathcal{R}\|_{g}^{2}
$$

(with $\|\overline{\mathbf{L}}\|_{g} \leq \bar{L}$ assumed to be bounded) has also been introduced and has the effect of removing $\|\overline{\mathbf{L}}\|_{g}$ from the equation for $\|\mathcal{R}\|_{g}$ as is consistent with zero order norm estimates. The turbulence model adopted in Moulden [3] placed:

$$
\mathbf{E}_{T}=\alpha_{e} \mathcal{R}+\alpha_{i} \operatorname{trace}(\mathcal{R}) \mathbf{I}, \quad \mathbf{D}_{F}=\alpha_{f} \mathcal{R}, \quad \mathbf{S}_{P}=\alpha_{s}[\mathcal{R}-\operatorname{trace}(\mathcal{R}) \mathbf{I} / 3]
$$

while the model for $\mathbf{D}_{P}$ was included in that for $\mathbf{D}_{F}$ (and was a simplified version of the model discussed in Lewellen [10]). The model satisfies the required Galilean covariance. Here, the set $\left\{\alpha_{e}, \alpha_{f}, \ldots\right\}$ are model constants (all greater than zero). The constant $\Lambda^{*}$ in the dynamical system above is given by the sum $\Lambda^{*}=2 \alpha_{s} / 3+$ $\alpha_{e}+\alpha_{f}+\alpha_{i}>0$. The Reynolds tensor norm decays like:

$$
\|\boldsymbol{R}\|_{g}^{2}(t) \leq \exp \left[-2\left(\nu\left(1+L_{R}^{2}\right)+\Lambda^{*}-2 \bar{L}\right) t\right]\|\mathcal{R}\|_{g}^{2}(0)
$$

provided that $\nu\left(1+L_{R}^{2}\right)+\Lambda^{*}>2 \bar{L}$. In the limit as $t \rightarrow \infty$, the mean velocity norm then decays as:

$$
|\mathbf{V}|_{g}^{2}(t) \leq \exp \left[-\nu L_{L}^{2} t / 2\right]|\mathbf{V}|_{g}^{2}(0)
$$

in the absence of a body force. Otherwise, $|\mathbf{V}|_{g}^{2} \rightarrow 2|\mathbf{f}|_{g}^{2} /\left(\nu L_{L}^{2}\right)^{2}$ as $t \rightarrow \infty$. Note that this is twice the value found in estimate (E1) for the instantaneous velocity norm $|\mathbf{v}|_{g}^{2}$. With the removal of the mean velocity gradient from the $\|\boldsymbol{R}\|_{g}$ equation any interaction of the Reynolds tensor with the mean motion is denied in this estimate. The estimate is not physically tenable. Hence first order norm closure, Moulden [3], must be adopted and an $\|\overline{\mathbf{L}}\|_{g}$ term retained in the $\|\boldsymbol{\mathcal { R }}\|_{g}$ equation. The limit $\|\mathcal{R}\|_{g} \rightarrow 0$ is understood to mean that $|\mathbf{u}| \rightarrow 0$ locally and that $|\mathbf{V}|_{g} \rightarrow$ $|\mathbf{v}|_{g}$. The finding above is another manifestation that the zero order norm closure estimate is unsatisfactory. The above has shown:

Lemma II. Zero order norm closure for this model does not provide a useful estimate for $|\mathbf{V}|_{g}$.

From Moulden [3], first order norm closure gave a limit for $|\mathbf{V}|_{g}$ that was consistent with estimate (E1) and was, in addition, linearly stable at the origin of phase space.

The present analysis does not carry epistemological content as far as the structure of the turbulence is concerned. The global norm quantities that enter the theory make no reference to the eddy sizes that contribute to the local motion. From the definition $|\mathbf{v}|_{g}^{2}=\int_{\mathcal{D}}\langle\mathbf{v}, \mathbf{v}\rangle d V$ a global norm contains energy from all wave numbers. It also contains energy from all points of the flow domain $\mathcal{D}$. Only the time evolution of the norm is determined from the theory. The work of Foias et al. [2] and Henshaw et al. [11] for example does, however, make reference to some physical aspects of the flow in terms of small and large scale motions. It is not clear at this point how such information should be included in a turbulence model - if, indeed, it is of importance so to do. Certainly, these two disparate flow components play very different roles in the flow development. As noted below, it is 
often necessary to modify turbulence models for application close to solid surfaces where eddy dissipation and production is a physically significant feature of the turbulence.

A comment can be made about the ubiquitous adoption of periodic boundary conditions in functional estimates. Such boundary conditions remove certain boundary integrals from the energy estimates. The flow region contained within the domain $\mathcal{D}$ is not totally arbitrary in the present theory since it can only contain solid boundaries if they have the required periodicity. This restriction is removed in Moulden [7].

\section{The " $k \sim \epsilon "$ model}

As a turbulence model, the $k \sim \epsilon$ model is less complex than methods based upon the full second moment equation (8). The six equations contained in (8) is reduced to two equations, one for $k$ and one for $\epsilon$. However, the resulting $k \sim \epsilon$ model is algebraically more complex due to the definitions of $P$ and the eddy viscosity $\nu_{\tau}$ in equations (15) and (16) below. That is ratios such as $\epsilon^{2} / k$ and $k^{2} / \epsilon$ arise in the model. Herein, only an examination of the system is presented with the full theory to appear in Moulden [7]. First, introduce the variables:

$$
\begin{array}{lr}
k=\operatorname{trace}(\boldsymbol{R}) / 2 & \text { (the turbulence kinetic energy) } \\
\left.\epsilon=\nu \mathcal{E}\left[\partial u_{i} / \partial x_{j}\right)^{2}\right] \equiv \nu \mathcal{E}\left[\operatorname{trace}\left(\mathbf{L}^{\prime} \mathbf{L}^{\prime T}\right] \quad\right. \text { (the turbulence dissipation) }
\end{array}
$$

both $k \geq 0$ and $\epsilon \geq 0$ are well defined physical quantities in the sense of being Galilean invariant and hence consistent with the Navier-Stokes equations.

Start the analysis with the adoption of a gradient transport model for the Reynolds tensor $\mathcal{R} \equiv \mathcal{E}(\mathbf{u} \otimes \mathbf{u})$ (as discussed in Bradshaw [12] for example). That is, adopt the form:

$$
\mathcal{R}=2 \nu_{\tau} \overline{\mathbf{D}}-2 k \mathbf{I} / 3
$$

which relates $\mathcal{R}$ to both the mean motion, via the symmetric part, $\overline{\mathbf{D}}$, of the mean velocity gradient, $\overline{\mathbf{L}}$, and the turbulence kinetic energy, $k(\mathbf{x}, t)$. The eddy viscosity $\nu_{\tau}=\nu_{\tau}(k, \epsilon)$ is taken to be a function of both the kinetic energy $k$ and the dissipation $\epsilon$. As in the Boussinesq model, $\mathcal{R}$ is related directly to the mean motion in equation (10), but this specification does not close the mean motion equations due to the inclusion of $k$ in equation (10). Equation (10) leads to the global norm inequality:

$$
\|\boldsymbol{R}\|_{g}^{2} \leq 2\left|\nu_{\tau}\right|_{g}^{2}\|\overline{\mathbf{L}}\|_{g}^{2}+4|k|_{g}^{2} / 3
$$

since the Korn inequality, see Horgan [13], shows that $\|\overline{\mathbf{D}}\|_{g}^{2}=\|\overline{\mathbf{L}}\|_{g}^{2} / 2$. Equation (11) may be compared with the inequality of Schumann [14] discussed in connection with the realizability conditions for the Reynolds tensor $\mathcal{R}$. Equation (10) further implies that:

$$
\operatorname{trace}\left(\mathcal{R} \overline{\mathbf{L}}^{T}\right)=\nu_{\tau} \operatorname{trace}(\overline{\mathbf{D}} \overline{\mathbf{D}}) \equiv \nu_{\tau}\|\overline{\mathbf{D}}\|^{2}=\nu_{\tau}\|\overline{\mathbf{L}}\|_{g}^{2} / 2
$$

and that:

$$
\langle\mathcal{R}, \overline{\mathbf{L}}\rangle_{g}=\left\langle\nu_{\tau} \overline{\mathbf{D}}, \overline{\mathbf{D}}\right\rangle_{g} \leq\left|\nu_{\tau}\right|\|\overline{\mathbf{D}}\|_{g}^{2} \equiv\left|\nu_{\tau}\right|\|\overline{\mathbf{L}}\|_{g}^{2} / 2
$$


from the Korn inequalities. Take the pair of equations:

$$
\begin{aligned}
& \frac{\partial k}{\partial t}+V_{j} \frac{\partial k}{\partial x_{j}}+Q=\frac{\partial}{\partial x_{j}}\left[\nu_{k} \frac{\partial k}{\partial x_{j}}\right] \\
& \frac{\partial \epsilon}{\partial t}+V_{j} \frac{\partial \epsilon}{\partial x_{j}}+P=\frac{\partial}{\partial x_{j}}\left[\nu_{\epsilon} \frac{\partial \epsilon}{\partial x_{j}}\right]
\end{aligned}
$$

as defining the model $k \sim \epsilon$ (see Wilcox [15] for example). This pair of equations represent a standard form of the model and needs modification near solid surfaces and as the motion decays to a quiescent state. In the above:

$$
P=c_{1} \epsilon^{2} / k+c_{2} \mathcal{R}_{i j} \bar{L}_{i j} \epsilon / k ; \quad Q=\epsilon+\mathcal{R}_{i j} \bar{L}_{i j}
$$

and:

$$
\nu_{k}=\nu+\nu_{\tau} ; \quad \nu_{\epsilon}=\nu+\nu_{\tau} / \sigma ; \quad \text { where } \nu_{\tau}=\alpha k^{2} / \epsilon
$$

the actual values of the constants $c_{1}, c_{2}, \sigma$ and $\alpha$ are not of importance at this point but do feature in the final stability result. It can also be noted that Lemma I suggests that $P$ and $Q$ are not uniquely defined since the transformation $\mathcal{R} \mapsto$ $\mathcal{R}+\mathcal{C}$ is permitted with $\operatorname{div}(\mathcal{C}) \equiv \mathbf{0}$. Hence equations (13) and (14) are not invariant under Lemma I. Equations $(15 \mathrm{a}, \mathrm{b})$ take on the local form:

$$
P=c_{1} \epsilon^{2} / k+\alpha c_{2} k\|\overline{\mathbf{D}}\|^{2} ; \quad Q=\epsilon+\nu_{\tau}\|\overline{\mathbf{D}}\|^{2}
$$

with a $\operatorname{trace}\left(\mathcal{C} \overline{\mathbf{L}}^{T}\right)$ non-uniqueness possible in $P$ and $Q$.

Look upon the system (13)-(16) as a set of equations for the four variables $k, \epsilon, P$ and $\nu_{\tau}$ which augment the mean motion equations (3) and (4). Since $P$ and $Q$ involve the norm $\|\overline{\mathbf{L}}\|_{g}^{2}$ there should be first order norm closure with equation (7) included for a consistent estimate. With estimate (11), equation (6a) for the mean velocity norm reduces to the differential inequality:

$$
\frac{\partial}{\partial t}|\mathbf{V}|_{g}^{2}+\frac{\nu L_{L}^{2}}{2}|\mathbf{V}|_{g}^{2} \leq \frac{2}{\nu^{3}}\left|\nu_{\tau}\right|_{g}^{2}+\frac{4}{3 \nu}|k|_{g}^{2}+\frac{1}{\nu L_{L}^{2}}|\mathbf{f}|_{g}^{2}
$$

when the Poincare inequality is employed. The evolution of the mean velocity norm, $|\mathbf{V}|_{g}^{2}$, depends upon the norms $\left|\nu_{\tau}\right|_{g}^{2}$ and $|k|_{g}^{2}$ as well as the body force norm. The norms of $k, \epsilon$ and $\nu_{\tau}$ are related by the inequality obtained from equation (16c):

$$
\alpha|k|_{g}^{2} \leq\left|\nu_{\tau}\right|_{g}|\epsilon|_{g} \leq\left[\left|\nu_{\tau}\right|_{g}^{2}+|\epsilon|_{g}^{2}\right] / 2
$$

while the norms of $P$ and $Q$ relate to other norms as:

$$
|P|_{g}^{2} \leq c_{1}\left|\mu_{\tau}\right|_{g}^{2}+\alpha c_{2}|k|_{g}^{2}\|\overline{\mathbf{L}}\|_{g}^{2} / 2 ; \quad|Q|_{g}^{2} \leq|\epsilon|_{g}^{2}+\left|\nu_{\tau}\right|_{g}^{2}\|\overline{\mathbf{L}}\|_{g}^{2} / 2
$$

if $\mu_{\tau}=\epsilon^{2} / k$ when $2|\epsilon|_{g}^{2} \leq|k|_{g}^{2}+\left|\mu_{\tau}\right|_{g}^{2}$. Illustrate the process for zero order norm closure when these inequalities reduce to:

$$
|P|_{g}^{2} \leq c_{1}\left|\mu_{\tau}\right|_{g}^{2}+\alpha c_{2}|k|_{g}^{2} \bar{L}^{2} / 2 ; \quad|Q|_{g}^{2} \leq|\epsilon|_{g}^{2}+\left|\nu_{\tau}\right|_{g}^{2} \bar{L}^{2} / 2
$$

using the bound $\|\overline{\mathbf{L}}\|_{g} \leq \bar{L}$ as above. 
Formally applying the Gronwall lemma to equation (17) (for which see Flavin and Rionero [16]) there is:

$$
|\mathbf{V}|_{g}^{2}(t) \leq|\mathbf{V}|_{g}^{2}(0) \exp \left[-\nu L_{L}^{2} t / 2\right]+\int_{0}^{t} F(t) \exp \left[-\nu L_{L}^{2} t / 2\right] d t
$$

if $F(t)$ denotes the right hand side of equation (17). The structure of equation (18) is the same as that for the instantaneous Navier-Stokes equations discussed earlier (from estimates (E1) and (E2)). However, the mean motion in estimate (18) decays as $\exp \left[-\nu L_{L}^{2} t / 2\right]$ whereas the instantaneous motion decays as $\exp \left[-\nu L_{v}^{2} t\right]$. The meaning here was mentioned above in relation to equation (10). The turbulent flow decay in estimate (18) has additional contributions from the norms $\left|\nu_{\tau}\right|_{g}$ and $|k|_{g}$ which relate to the turbulent fluctuations. In the case where the body force is independent of time and the norm $|k|_{g}$ decays with time, the limit $t \rightarrow \infty$ in equation (18) gives the form:

$$
|\mathbf{V}|_{g}^{2}(t) \leq 2|\mathbf{f}|_{g}^{2} /\left(\nu L_{L}^{2}\right)^{2} \quad \text { as } t \rightarrow \infty
$$

which is twice the body force contribution found for the instantaneous equations. It is again suggested that this can be attributed to the adoption of a zero order norm closure in the equations without appeal to equation (7) for the mean velocity gradient norm.

The $k \sim \epsilon$ model provides an evolution inequality for both $|k|_{g}^{2}$ and $|\epsilon|_{g}^{2}$ from equations (13) and (14).

$$
\begin{gathered}
\frac{\partial|k|_{g}^{2}}{\partial t}+(\nu+n) L_{k}^{2}|k|_{g}^{2} \leq \frac{1}{4(\nu+n) L_{k}^{2}}|Q|_{g}^{2} \\
\frac{\partial|k|_{g}^{2}}{\partial t}+(\nu+n / \sigma) L_{\epsilon}^{2}|\epsilon|_{g}^{2} \leq \frac{1}{4(\nu+n / \sigma) L_{\epsilon}^{2}}|P|_{g}^{2}
\end{gathered}
$$

where the bounded constant $n=\left.\nu_{\tau}\right|_{\max }$ on $\mathcal{D}$ has been introduced. From the definitions in equation (15), the norms $|P|_{g}^{2}$ and $|Q|_{g}^{2}$ are related by the inequality:

$$
\left(2 c_{1}-3 c_{2}\right)|\epsilon|_{g}^{2} \leq c_{2}|Q|_{g}^{2}+|P|_{g}^{2}+|k|_{g}^{2}
$$

The model as treated herein is not intended to apply to wall bounded flows (some of the properties of which are discussed in Patel et al. [17] as well as in Nagano and Tagawa [18]). In particular, $k \rightarrow 0$ at a solid surface but $\epsilon$ does not: hence $\mu_{\tau}$ is not well defined. Such wall bounded flows are examined in Moulden [7].

A system of equations has been obtained for the four unknown norm variables in the $k \sim \epsilon$ model. In actual estimates, it is required that values be given to the Poincaré constants $\left\{L_{L} ; L_{k} ; \cdots\right\}$. It is well known, see Moulden [19] for actual computations in a specific case, that the values of these constants can vary over a range of several orders of magnitude. This happenstance calls into question the actual numerical values adopted to determine the stability of the dynamical system. 
The dynamical system of interest is constructed from equations (17), (19) and (20):

$$
\frac{\partial}{\partial t}\left(\begin{array}{c}
|\mathbf{V}|_{g}^{2} \\
|k|_{g}^{2} \\
|\epsilon|_{g}^{2}
\end{array}\right) \leq\left(\begin{array}{c}
-\nu L_{L}^{2}|\mathbf{V}|_{g}^{2} / 2+2\left|\nu_{\tau}\right|_{g}^{2} / \nu^{3}+4|k|_{g}^{2} /(3 \nu)+|\mathbf{f}|_{g}^{2} /\left(\nu L_{L}^{2}\right) \\
-(\nu+n) L_{k}^{2}|k|_{g}^{2}+\left[|\epsilon|_{g}^{2}+\left|\nu_{\tau}\right|_{g}^{2} \bar{L}^{2}\right] /\left(8(\nu+n) L_{k}^{2}\right) \\
-\nu^{*} L_{\epsilon}^{2}|\epsilon|_{g}^{2}+\left[c_{1}\left|\mu_{\tau}\right|_{g}^{2}+\nu c_{2}|k|_{g}^{2} \bar{L}^{2}\right] /\left(8 \nu^{*} L_{\epsilon}^{2}\right)
\end{array}\right)
$$

where $\nu^{*}=\nu+n / \sigma$. Robinson [20] discusses properties of dynamical systems which apply to the present situation. The fixed points of the corresponding differential system can be established. By definition tenable solutions cannot have negative values for the norms involved. Hence the fixed points (where the right hand side vanishes) defines an equilibrium state for the motion. Note that the limit $|k|_{g}^{2} \rightarrow 0$ and $|\epsilon|_{g}^{2} \rightarrow 0$ cannot be taken for this model for reasons discussed above. There is however:

Lemma III. The $k \sim \epsilon$ model introduced above has a fixed point where the mean velocity norm, for example, has the value:

$$
|\mathbf{V}|_{g}^{2}=\frac{2}{\nu L_{L}^{2}}\left[\frac{2}{\nu^{3}}\left|\nu_{\tau}\right|_{g}^{2}+\frac{4}{3 \nu}|k|_{g}^{2}+\frac{1}{\nu L_{L}^{2}}|\mathbf{f}|_{g}^{2}\right]
$$

in terms of turbulence quantities and the body force norm. This fixed point is linearly stable.

to complete the discussion of this model.

As a final note to this section there have been many modifications to the $k \sim \epsilon$ model and Nagano and Tagawa [18] being one such addition to allow for near wall effects. Such models call for additional functions, $f_{\nu}, f_{\epsilon}$ and $f_{u}$, say, which allow for damping close to a solid wall. With these functions $P$ is modified to:

$$
P=c_{1} f_{\epsilon} \epsilon^{2} / k+c_{2} \mathcal{R}_{i j} \bar{L}_{i j} f_{u} \epsilon / k
$$

while the eddy viscosity coefficient is changed to the form $\nu_{\tau}=\alpha f_{\nu} k^{2} / \epsilon$. The free turbulence limit requires a separate treatment.

\section{Final comments}

Some comments have been made concerning the structure of norm estimates and their application to both a second moment model and to the $k \sim \epsilon$ turbulence model. Without a body force present it was found that the second moment model predicts that the motion will decay to the origin of phase space. In the case of the $k \sim \epsilon$ model, there was a limitation to fixed points in a uniform flow where $\partial|\mathbf{V}|_{g}^{2} / \partial t \equiv 0$. That flow is linearly stable.

\section{References}

[1] Gatski, T. B: Constitutive equations for turbulent flows, Th. and Comp. Fluid Dyn., 18, No 5, pp 345-369, 2004. 
[2] Foias, C. Manley, O. and Temam, R: Modeling of the interaction of small and large eddies in two-dimensional turbulence, Math. Modeling and Num. Anal., 22, pp 93-118, 1988.

[3] Moulden. T. H: Estimates for turbulence models, Int. J. Pure and Appl. Math., 43, pp 551-562, 2008.

[4] Speziale, C. G: Invariance of turbulence closure models, Phys. Fluids, 22, pp 1033-1037, 1979.

[5] Hanjalić, K: Second-moment turbulence closures for CFD: needs and prospects, IJCFD, 12, pp 67-97, [1999].

[6] Gurtin, M. E: An Introduction to Continuum Mechanics, Academic Press, New York, 1981.

[7] Moulden, T. H: Viscous Fluid Motion, Text in preparation.

[8] Seregin, G: Local regularity of the Navier Stokes equations, In: Friedlander, S. and Serre, D. (Eds). "Handbook of Mathematical Fluid Mechanics”, 4, pp 159-200, North Holland, Amsterdam, 2007.

[9] Bradshaw, P: Turbulence: the chief outstanding difficulty of our subject, Exp. in Fluids., 16, pp 203-216, 1994.

[10] Lewellen, W. S: Use of invariant modeling, In: Frost, W. and Moulden, T. H. (Eds). Handbook of Turbulence, Plenum Press, New York, pp 237-280, 1977.

[11] Henshaw, W. D, Kreiss, H. O. and Reyna, L. G: Smallest scale estimates for the Navier-Stokes equations for incompressible fluids, Arch. Rat. Mech. Anal., 112, pp 21-44, 1990.

[12] Bradshaw, P. (Ed): Turbulence, 2nd Edition. Springer Verlag, Berlin, 1978.

[13] Horgan, C. O: Korn's inequalities and their application in continuum mechanics, SIAM Review, 37, pp 491-511, 1995.

[14] Schumann, U: Realizability of Reynolds-stress turbulence models, Phys. Fluids, 20, pp 721-725, 1977.

[15] Wilcox, D. C: Turbulence Models for CFD, DCW Industries, La Cafiada, 1998.

[16] Flavin, J. N. and Rionero, S: Qualitative Estimates for Partial Differential Equations, CRC Press, Boco Raton, 1996.

[17] Patel, V. C., Rodi, W. and Scheuerer, G: Turbulence models for near-wall and low Reynolds number flows: a review, AIAA J., 23, pp 1308-1319, 1985.

[18] Nagano, Y. and Tagawa, M: An improved $k \sim \epsilon$ model for boundary layer flows, J. Fluids Eng., 112, pp 33-39, 1990.

[19] Moulden, T. H: On the structure of Burgers' turbulence, 5th International Conference of Applied Mathematics, Plovdiv, Bulgaria, 2008.

[20] Robinson, J. C: Infinite Dimensional Dynamical Systems, Cambridge University Press, Cambridge, 2001. 